\title{
Review on Charmonium Production
}

\author{
Katja Krüger \\ Ruprecht-Karls-Universität Heidelberg - Kirchhoff-Institut für Physik \\ Im Neuenheimer Feld 227, 69120 Heidelberg - Germany
}

\begin{abstract}
A brief overview on recent results of charmonium production in high-energy protonproton, electron-proton and electron-positron collisions is presented. Emphasis is given to QCD dominated production mechanisms that allow a study of the interplay between perturbative and non-perturbative effects.
\end{abstract}

\section{Introduction}

Charmonium production in high-energy collisions [1] can be described by the perturbative production of a $c \bar{c}$ pair followed by the non-perturbative transition to the charmonium state. This transition can be modelled in different ways. An attractive approach is Non-Relativistic QCD (NRQCD) [2] which uses a factorization ansatz and takes into account all possible colour and angular momentum states for the $c \bar{c}$ pair. The transition to the charmonium state proceeds via soft gluon emission and is described by long-distance matrix elements which are universal but have to be determined from data. In the older Colour Singlet Model (CSM) [3] the $c \bar{c}$ pair has to be produced perturbatively in the same quantum state as the charmonium. For the $\psi$ states the transition rate is determined from the leptonic decay widths. The production of $P$ wave states cannot be predicted since it leads to divergences in the calculation [4].

Experimental results on charmonium production are dominated by $J / \psi$ mesons due to the large cross section and the large branching fraction to leptons. The main disadvantage is the feeddown from $B$ mesons and higher charmonium states. The feeddown is reduced for the $\psi(2 s)$ meson, which is otherwise very similar. The $P$ wave states $\chi_{c 0}, \chi_{c 1}$ and $\chi_{c 2}$ offer the possibility to study a different angular momentum state.

\section{Charmonium production at the Tevatron}

The prompt $J / \psi$ and $\psi(2 s)$ production cross section at the Tevatron is more than an order of magnitude larger than expected by the CSM in leading order (LO). Only recently calculations [5] in next-to-leading order (NLO) have been performed which improve the data description showing a larger cross section and a milder drop with the transverse momentum. The difference between the data and the CSM can be explained by additional colour-octet contributions in the context of NRQCD. In this approach the non-perturbative parameters can be extracted from the transverse momentum spectrum. A very decisive test of NRQCD is the measurement of the polarization of the $J / \psi$ and $\psi(2 s)$ mesons. Polarization studies have the advantage of not being sensitive to the absolute rates, but need a huge amount of statistics to provide significant results. CDF Results [6] from Tevatron Run I indicated a transverse polarization (positive $\alpha$ ) at large transverse momenta as expected from the gluon fragmentation contribution in NRQCD calculations, while the new measurement [7] with higher statistics from Run II shows a small longitudinal polarization in this region (Fig. 1 left). Similar, but statistically less significant results are obtained for prompt $\psi(2 s)$ production. 

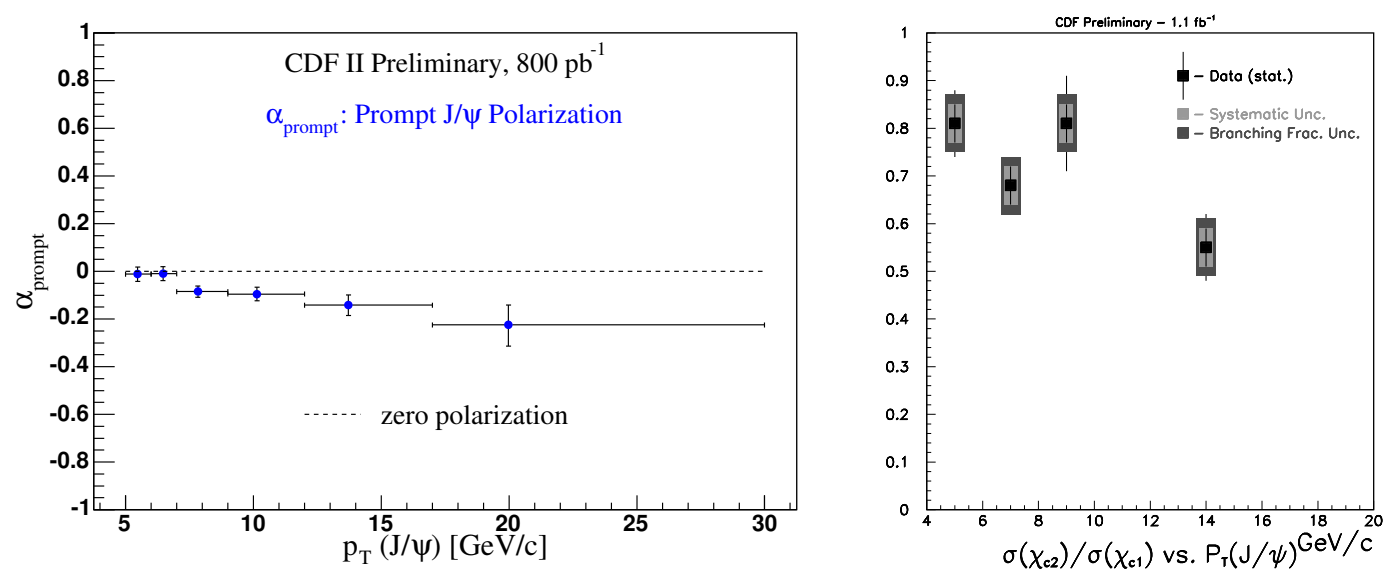

Figure 1: Charmonium production at the Tevatron: $J / \psi$ polarization (left) and ratio of prompt production of $\chi_{c 2}$ to $\chi_{c 1}$ (right).

The CDF collaboration presented a measurement [8] of prompt $\chi_{c}$ production via their decays into $J / \psi \gamma$. The photon is reconstructed through conversion, which gives a mass resolution sufficient for resolving the different $\chi_{c}$ states. The result is a ratio of $\chi_{c 2} / \chi_{c 1}=$ $0.70 \pm 0.04$ (stat. $) \pm 0.04$ (sys.) \pm 0.06 (branching fraction) with no significant dependence on the transverse momentum (Fig. 1 right) and no signal for the $\chi_{c 0}$. This is in contradiction to the expectation from NRQCD, where the ratios should be $\chi_{c 2}: \chi_{c 1}: \chi_{c 0}=5: 3: 1$ according to simple spin counting rules.

\section{Charmonium production at HERA}

In electron-proton collisions two kinematic regimes are distinguished according to the virtuality $Q^{2}$ of the exchanged photon. In the photoproduction region, where $Q^{2} \approx 0$, predictions for the CSM are available at next-to-leading order, while in electroproduction at larger $Q^{2}$ only leading order calculations exist. Recently new experimental results from the HERA II phase with improved statistics allow more detailed comparisons to the predictions.

\subsection{Photoproduction}

In general the photoproduction of $J / \psi$ mesons at HERA $[9,10]$ is well described by the NLO predictions [13] in the CSM, while LO NRQCD predictions have difficulties to describe the distribution of $z$, the relative energy transfer from the photon to the $J / \psi$ meson. For the $J / \psi$ polarization [11], where only LO

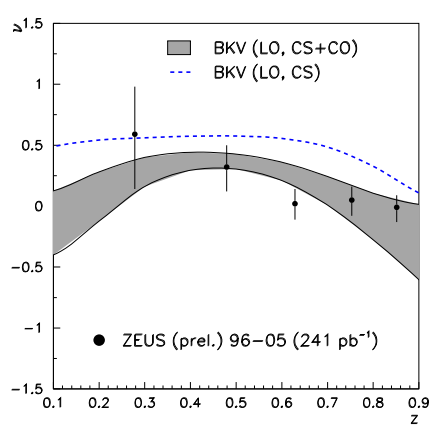

Figure 2: $J / \psi$ polarization in photoproduction at HERA in comparison with LO CSM (dashed line) and NRQCD (grey band) calculations. 
similar, some deviations of the CSM are seen at large

$z$, while the NRQCD calculation agrees better with the data (Fig. 2).

\subsection{Electroproduction}

In $J / \psi$ electroproduction $Q^{2}$ provides an additional hard scale which should lead to a better control of the theoretical predictions. In general the LO predictions in the CSM underestimate the data by a factor $\sim 2-3$ and the transverse momentum distribution falls too steeply, while NRQCD can describe the normalization, but fails for the $z$ distribution. For a new double differential measurement [12] in $z$ and the transverse momentum in the photon-proton centre-of-mass frame (Fig. 3) LO CSM Monte Carlo models agree well with data in shape and show, that additional colour octet contributions must be small or very similar in shape to the colour singlet

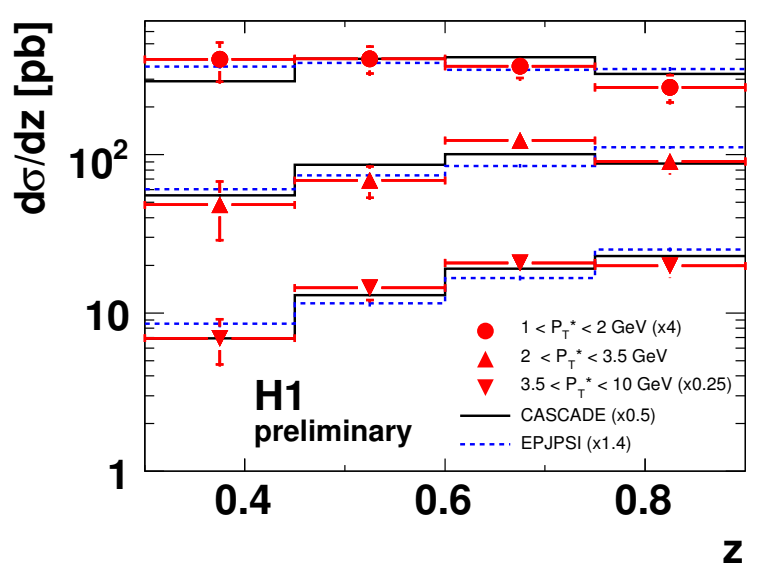

Figure 3: $J / \psi$ electroproduction at HERA: double differential cross section compared to two LO CSM Monte Carlo predictions. terms.

\section{Charmonium production in electron-positron-annihilations}

For the $J / \psi$ production at the $\Upsilon(4 S)$ resonance in electron-positron collisions the momentum spectrum in the centre-of-mass frame (Fig. 4) as measured by the BaBar [14] and Belle [15] collaborations provides a test of the production mechanism. LO NRQCD calculations predict an enhancement of the cross section at large momentum which is not observed in the data, while the CSM underestimates the cross section. Including perturbative and non-perturbative resummations in the NRQCD calculation, the data can be described in shape and normalization [16].

\section{Conclusions}

Charmonium production in high-energy collisions is a very active field of research. A wealth of experimental results is available and more data from the full statistics of the second running phase of the Tevatron and the HERA colliders are expected soon. All theoretical calculations fail to describe some of the measurements, so a coherent picture is still lacking.

\section{References}

[1] Slides: http://indico . cern. ch/contributionDisplay . py? contribId=206\&sessionId=5\&conf Id=9499 

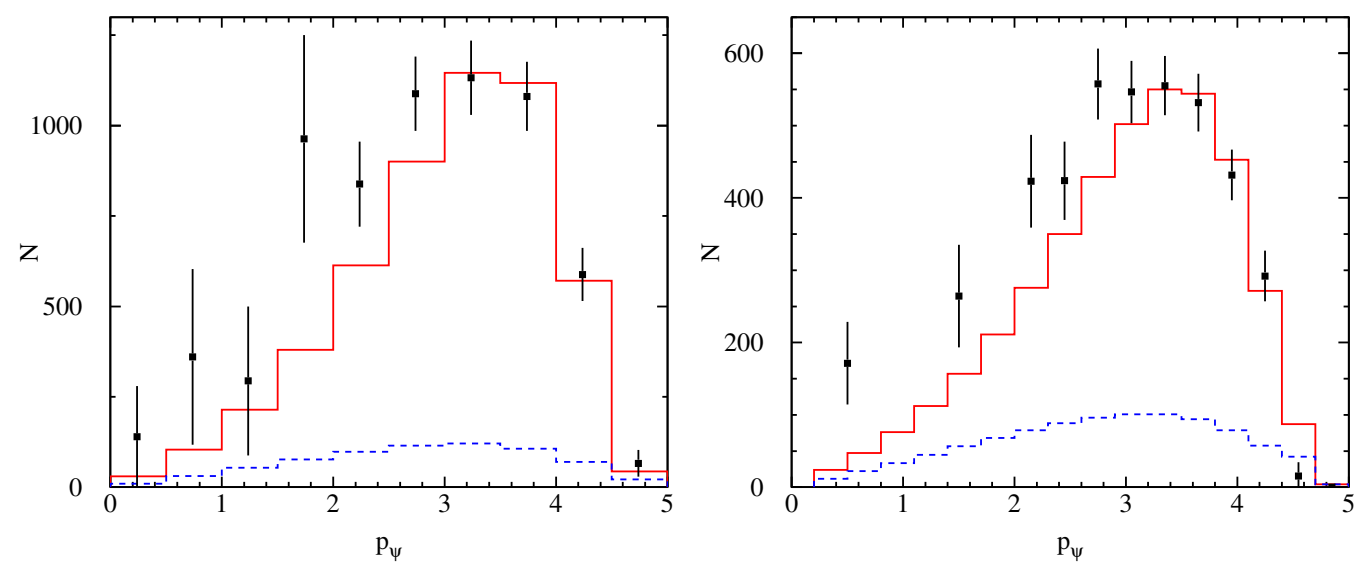

Figure 4: Momentum spectrum of $J / \psi$ mesons produced in $e^{+} e^{-}$-annihilations at the $\Upsilon(4 S)$ measured by BaBar (left) and Belle (right) compared to predictions in the CSM (dashed line) and in NRQCD including perturbative and non-perturbative resummations (solid line).

[2] G. T. Bodwin, E. Braaten and G. P. Lepage, Phys. Rev. D 51 (1995) 1125, Erratum ibid. D 55 (1997) 5853 [arXiv:hep-ph/9407339]

[3] E. L. Berger and D. L. Jones, Phys. Rev. D 23 (1981) 1521;

R. Baier and R. Rückl, Phys. Lett. B 102 (1981) 364

[4] M. Krämer, Prog. Part. Nucl. Phys. 47, 141 (2001) [arXiv:hep-ph/0106120]

[5] J. Campbell, F. Maltoni and F. Tramontano, arXiv:hep-ph/0703113

[6] A. A. Affolder et al. [CDF Collaboration], Phys. Rev. Lett. 85 (2000) 2886 [arXiv:hep-ex/0004027]

[7] CDF Collaboration, "Measurement of the $J / \psi$ Spin Alignment in $\bar{p} p$ Collisions at $\sqrt{s}=1.96 \mathrm{TeV}$ ", CDF note 8212 (2006)

[8] A. Abulencia et al., arXiv:hep-ex/0703028

[9] C. Adloff et al. [H1 Collaboration], Eur. Phys. J. C 25 (2002) 25 [arXiv:hep-ex/0205064]

[10] S. Chekanov et al. [ZEUS Collaboration], Eur. Phys. J. C 27 (2003) 173 [arXiv:hep-ex/0211011]

[11] ZEUS Collaboration, "Measurement of $J / \psi$ helicity distributions in inelastic photoproduction at HERA", ZEUS-prel-06-015 (2006), Contributed Paper to ICHEP06

[12] H1 Collaboration, Slides: http://indico. cern. ch/contributionDisplay. py? contribId=78\&sessionId=13\&conf Id=9499

[13] M. Krämer, J. Zunft, J. Steegborn and P. M. Zerwas, Phys. Lett. B 348 (1995) 657 [arXiv:hep$\mathrm{ph} / 9411372]$

[14] B. Aubert et al. [BABAR Collaboration], Phys. Rev. Lett. 87 (2001) 162002 [arXiv:hep-ex/0106044]

[15] K. Abe et al. [BELLE Collaboration], Phys. Rev. Lett. 88 (2002) 052001 [arXiv:hep-ex/0110012]

[16] S. Fleming, A. K. Leibovich and T. Mehen, Phys. Rev. D 68 (2003) 094011 [arXiv:hep-ph/0306139] 